\title{
Winding a nanotube from black phosphorus nanoribbon onto a CNT at low temperature: A molecular dynamics study
}

\author{
Kun Cai ${ }^{\text {a,b }}$, Lingnan Liu ${ }^{\text {a }}$, Jiao Shi ${ }^{\text {a }}$, Qing H. Qin ${ }^{\text {b,* }}$ \\ ${ }^{a}$ College of Water Resources and Architectural Engineering, Northwest A\&F University, Yangling 712100, China \\ ${ }^{b}$ Research School of Engineering, The Australian National University, ACT 2601, Australia
}

\section{H I G H L I G H T S}

- Fabricating a black phosphorene (BP) nanotube from a BP nanoribbon is studied.

- Winding a BP ribbon on a CNT may form a perfect BP nanotube at low temperature.

- At T $\leq 50 \mathrm{~K}$, the BP nanoribbon with perfect length can form a BP nanotube on the CNT.

- A BP ribbon longer than perfect length cannot form a nanotube on the CNT.

\section{A R T I C L E I N F O}

\section{Article history:}

Received 3 December 2016

Received in revised form 24 February 2017

Accepted 26 February 2017

Available online 28 February 2017

\section{Keywords:}

Black phosphorus

Nanotube

Carbon nanotube

Molecular dynamics

\section{G R A P H I C A L A B S T R A C T}

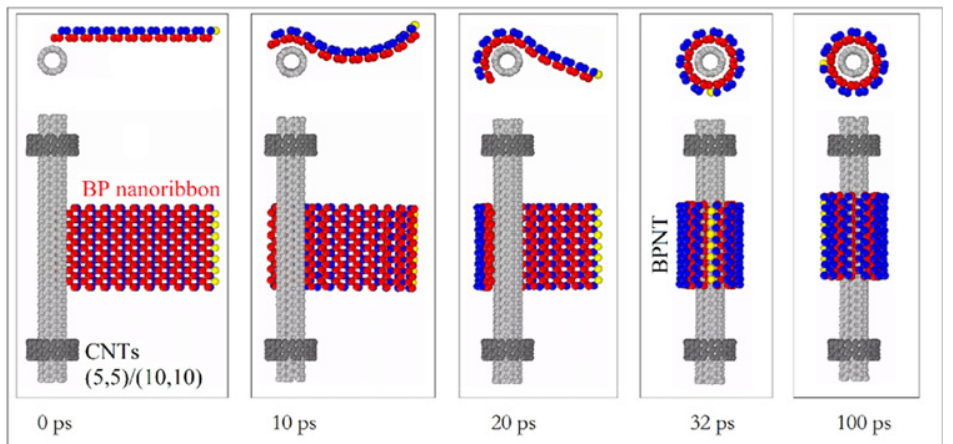

\begin{abstract}
A B S T R A C T
Black phosphorus (BP) has become a popular two dimensional semiconducting material. Investigation of a BP nanotube, theoretically and experimentally, is therefore a hot topic attracting many recent researchers. In this paper, a nanotube system is proposed by placing a BP ribbon near a carbon nanotube (CNT) to form a BP nanotube. The system is formed based on the following two aspects. The first is that the CNT has a perfect surface that provides only van der Waals attraction to the BP ribbon. The BP ribbon does not damage the CNT. The second aspect is that a BP ribbon can feature a large curvature without breaks of the phosphorus-phosphorus bonds in it. If the length of the BP ribbon is well chosen to be a 'perfect length', the ribbon can form a perfect BP nanotube winding on a CNT at very low temperature. At higher temperature, e.g., $\geq 50 \mathrm{~K}$, it is difficult to obtain a perfect $\mathrm{BP}$ nanotube. With a BP ribbon whose length is greater than the perfect length, it is difficult to create a perfect $\mathrm{BP}$ nanotube even at ultralow temperature.
\end{abstract}

(c) 2017 Elsevier Ltd. All rights reserved.

\section{Introduction}

With the rapid development of nanotechnology, low dimensional nano materials can be obtained easily and their physical properties studied profoundly. Typical low dimensional materials include

\footnotetext{
* Corresponding author at: Research School of Engineering, The Australian National University, Building 32, Acton 2601, Australia.

E-mail address: qinghua.qin@anu.edu.au (Q.H. Qin).
}

graphene [1-5], $\mathrm{MoS}_{2}$ [6,7], BN [8,9], and black phosphorus (BP) [10, 11], carbon nanotubes [12-16]. Although the electrical properties of BP have been reported for a few decades [17,18], it has not become popular until recently due to the works of Refs $[10,19,20]$. In these works, the electric field effect of a field effect transistor made from fewlayered BP was studied and excellent transistor performances of such 2D material at room temperature were obtained. There are several allotropic forms of phosphorus. Unlike carbon allotropes that include 3D diamond, 2D graphene, and 1D carbon nanotube (CNT), no nanotube 
(a)

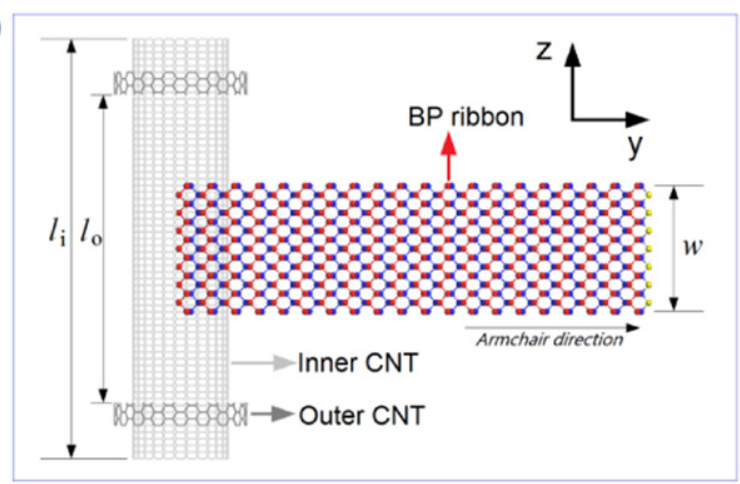

(b)

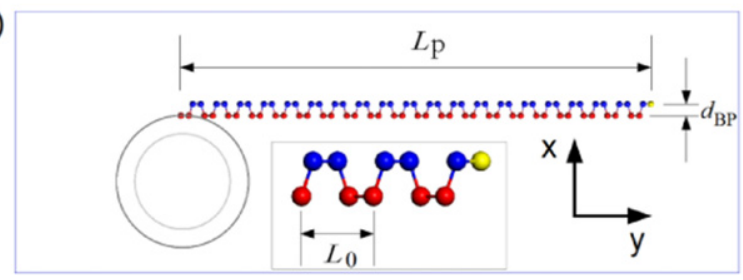

Fig. 1. Schematic geometric model (in $x-y-z$ coordinate system) of a nano-system consisting of a double walled carbon nanotube and a black phosphorus ribbon. (a) Top view of the system; (b) axial view of the system. $l_{\mathrm{i}}$ is the length of the inner tube. $l_{\mathrm{o}}$ is the axial distance between the two outer tubes. The width of BP ribbon $w$ is $\sim 2.32 \mathrm{~nm}$, and is identical in all models. $L_{0}$ is the unit length of BP ribbon along the armchair direction, here $\sim 0.44 \mathrm{~nm}$. $L_{\mathrm{p}}$ is the perfect length of BP ribbon along the armchair direction. The internal edges of the two outer tubes are hydrogenated to avoid strong interaction between the CNT and BP ribbon. The atoms on the outer face of BP ribbon are colored blue and those on the inner face are red. The phosphorus atoms on the edge that are the furthest from the inner CNT are yellow and are used to judge the generation of new phosphorus-phosphorus (P-P) bonds. For BP ribbon, the $\mathrm{y}$-direction is the armchair direction and the $\mathrm{z}$-axis is along the zigzag direction.

from a phosphorus allotrope has ever been discovered in laboratory. Among the phosphorus allotropes, BP is the most stable, as each phosphorus atom in a single-layered BP is covalently bonded with three adjacent phosphorus atoms, and all the three valence electrons of each phosphorus atom are occupied. Compared to $2 \mathrm{sp}^{2}$ or $2 \mathrm{sp}^{3}$ orbitals in carbon allotropes, the $3 \mathrm{sp}^{3}$ hybrid orbital based phosphorusphosphorus $(\mathrm{P}-\mathrm{P})$ bond is much weaker, which leads to the lower Young's modulus [21,22] and lower strength [23] of BP than those properties in graphene or diamond. Besides, at the pressure of 1 bar, BP behaves unstably even at $600 \mathrm{~K}$, which is much lower than the equivalent levels in graphene or diamond. Consequently, it must be a challenge to form a phosphorus nanotube using a self-assembly method, such as a chemical vapor deposition approach. Nevertheless, this difficulty seems not to reduce the curiosity of theoretical researcher exploring the fascinating 1D material, a BP nanotube. For example, Guo et al. [24] investigated the band gap of BP nanotubes by first principle calculations, and stated that the gap for BP nanotubes depended on such factors as the radius of the tube, chirality, and axial strain. It is well known that the outer surface of a BP nanotube is under a tensile state while the inner surface is compressed. Considering the weakness of $\mathrm{P}-\mathrm{P}$ bonds in a BP nanotube, Cai et al. [25] recently investigated the stability of both armchair and zigzag BP nanotubes at finite temperatures. Their results indicated that the radius of a stable armchair BP nanotube can be far less than that of a stable zigzag BP nanotube at the same temperature. Further, they [26] discussed the structural stability of armchair $\mathrm{BP}$ nanotubes under uniaxial compression along the tube axis. They found that the tubes entered buckling states when the axial strain was around 5\%, indicating that the deformation of the BP nanotube was under linear elastic deformation before buckling.

Before successful fabrication of a BP nanotube, the preceding research work has been limited to theoretical aspects only. The need to find an approach to fabricate a BP nanotube, either theoretically or experimentally, is becoming urgent. Considering state-of-the-art nanotechnology, the self-assembly of a nanotube directly from phosphorus atoms is not feasible. Besides, the weakness of $\mathrm{P}-\mathrm{P}$ bonds in $\mathrm{BP}$ must be confronted in fabrication. Hence, forming a nanotube by winding a $\mathrm{BP}$ nanoribbon seems to be feasible [27-30]. Simultaneously, considering the level of surface energy of a perfect CNT, it provides only enough van der Waals force to attract the BP ribbon but not damage it. In this study, molecular dynamics simulations are used to verify the prediction of forming a BP nanotube from a BP nanoribbon near a CNT.

\section{Models and methodology}

To reveal the influence of such factors as the chirality of CNTs, the radius of CNTs, and the environmental temperature on the winding process of BP ribbon, we build 3 groups containing 9 models of the system, as shown in Fig. 1. The essential parameters of the models are listed in Table 1.

The nano-system shown in Fig. 1 has three types of atoms: phosphorus, carbon, and hydrogen atoms. The interactions among the atoms are evaluated using three different potential functions. Briefly, the interaction among phosphorus atoms in BP ribbon is determined by the Stillinger-Weber [31] potential, with new parameters developed by Jiang [32,33]. AIREBO potential [34] is employed to estimate the interaction among carbon and/or hydrogen atoms in a CNT. The interactions among atoms on different components, e.g., inner CNT, outer CNT and BP ribbon, are evaluated through a 12-6 Lennard-Jones potential [35] using the constants listed in Table 2 . The interactions between phosphorus and hydrogen atoms are neglected in the present simulations.

It is known that attraction between BP ribbon and inner CNT can change their initial configurations from those shown in Fig. 1. If the interaction between the two components is strong enough, the BP ribbon will curve and cover the inner CNT to reduce the surface energy. To estimate the stable configurations of BP ribbon after a period of attraction by an inner CNT, we perform simulations in the Large-scale Atomic/Molecular Massively Parallel Simulator (LAMMPS $[36,37]$ ) package. The whole system is placed in a canonical NVT ensemble with specified temperature $\mathrm{T}(8 \mathrm{~K}, 50 \mathrm{~K}, 100 \mathrm{~K}$, and $150 \mathrm{~K}$ ) for a period of no more than 1000 ps. The positions in the system are updated every 0.001 ps. In the present study, the temperature is evaluated according to the state

Table 1

Parameters of the nano-system in Fig. 1, and number of carbon $(\mathrm{C})$, phosphorous $(\mathrm{P})$ and hydrogen $(\mathrm{H})$ atoms.

\begin{tabular}{|c|c|c|c|c|c|c|}
\hline Model no. & CNTs & Number of atoms on CNTs and BP ribbon & Perimeter of outer CNT & $l_{\mathrm{i}}(\mathrm{nm})$ & $l_{\mathrm{o}}(\mathrm{nm})$ & $L \mathrm{p}(\mathrm{nm})$ \\
\hline 1 & $(5,5) /(10,10)$ & $640 \mathrm{C}+160 \mathrm{C}+40 \mathrm{H}+330 \mathrm{P}$ & 4.26 & 7.75 & 5.58 & 4.75 \\
\hline 2 & $(6,4) /(11,9)$ & $608 \mathrm{C}+160 \mathrm{C}+40 \mathrm{H}+330 \mathrm{P}$ & 4.27 & 7.40 & 5.67 & 4.75 \\
\hline 3 & $(8,0) /(17,0)$ & $608 \mathrm{C}+272 \mathrm{C}+34 \mathrm{H}+330 \mathrm{P}$ & 4.18 & 7.95 & 5.55 & 4.75 \\
\hline 4 & $(9,9) /(14,14)$ & $1152 \mathrm{C}+224 \mathrm{C}+56 \mathrm{H}+450 \mathrm{P}$ & 5.96 & 7.75 & 5.58 & 6.49 \\
\hline 5 & $(13,4) /(23,2)$ & $1614 \mathrm{C}+410 \mathrm{C}+50 \mathrm{H}+450 \mathrm{P}$ & 5.92 & 9.60 & 5.79 & 6.49 \\
\hline 6 & $(15,0) /(24,0)$ & $1140 \mathrm{C}+384 \mathrm{C}+48 \mathrm{H}+450 \mathrm{P}$ & 5.90 & 7.95 & 5.55 & 6.49 \\
\hline 7 & $(13,13) /(18,18)$ & $1664 \mathrm{C}+288 \mathrm{C}+72 \mathrm{H}+570 \mathrm{P}$ & 7.67 & 7.75 & 5.58 & 8.68 \\
\hline 8 & $(14,12) /(19,17)$ & $1736 \mathrm{C}+304 \mathrm{C}+72 \mathrm{H}+570 \mathrm{P}$ & 7.67 & 8.10 & 5.68 & 8.68 \\
\hline 9 & $(22,0) /(31,0)$ & $1672 \mathrm{C}+496 \mathrm{C}+62 \mathrm{H}+570 \mathrm{P}$ & 7.62 & 7.95 & 5.55 & 8.68 \\
\hline
\end{tabular}


Table 2

Parameters in the L-J potential function $V\left(r_{\mathrm{ij}}\right)=4 \varepsilon\left[\left(\sigma / r_{\mathrm{ij}}\right)^{12}-\left(\sigma / r_{\mathrm{ij}}\right)^{6}\right]$ between two of C, P, and $\mathrm{H}$ atoms.

\begin{tabular}{llll}
\hline Atom $\mathrm{i}$ & Atom $\mathrm{j}$ & $\sigma(\AA)$ & $\varepsilon(\mathrm{eV})$ \\
\hline $\mathrm{P}$ & $\mathrm{C}$ & 3.4225 & 0.006878 \\
$\mathrm{P}$ & $\mathrm{P}$ & 3.438 & 0.015940 \\
$\mathrm{C}$ & $\mathrm{C}$ & 3.400 & 0.002844 \\
$\mathrm{C}$ & $\mathrm{H}$ & 3.025 & 0.002065 \\
$\mathrm{H}$ & $\mathrm{H}$ & 2.650 & 0.001499 \\
\hline
\end{tabular}

equation $E_{\mathrm{K}}=3 N_{\mathrm{a}} k_{\mathrm{B}} \mathrm{T} / 2$, where $E_{\mathrm{K}}$ represents the sum of the average kinetic energy of all the atoms in system, $N_{\mathrm{a}}$ is the number of atoms involved in the temperature calculation, $k_{\mathrm{B}}$ is the Boltzmann constant. The temperature is modified using a Nosé-Hoover thermostat [38].

During interactions among the components in such a system, the potential energy of the system may change due to variation of configuration. Three major factors influencing the variation of potential energy $(\triangle \mathrm{PE})$ of the system are attributed to changes of configuration of the system. The first factor is the deformation-based elastic potential energy of the BP ribbon. It depends on the radius of curvature of the wound $\mathrm{BP}$ ribbon. According to the theory of elasticity, the value of $\triangle \mathrm{PE}$ can be calculated according to the equation:

$\Pi_{\text {Deform }}=\int_{0}^{L_{\mathrm{p}}} \frac{\mathrm{E} \cdot \mathrm{I}}{2[\rho s]^{2}} \mathrm{~d} s$

where $\mathrm{E}$ is the equivalent Young's modulus of the BP ribbon along the armchair direction, i.e., $44 \mathrm{GPa}$ [22]. I is the inertia moment of the $\mathrm{X}-\mathrm{z}$ cross-section of the ribbon along its neutral layer. $\rho$ is the radius of the curvature of homogenized BP ribbon, which is a constant only when the ribbon perfectly covers the inner CNT. That constant is $\sim\left(r+\sigma_{\mathrm{C}-}\right.$ $\left.\mathrm{P}+0.5 d_{\mathrm{BP}}\right)$, with $r$ the radius of inner CNT, $\sigma_{\mathrm{C}-\mathrm{P}}$ the equilibrium distance between $\mathrm{BP}$ ribbon and CNT, and $d_{\mathrm{BP}}$ the distance between two surfaces of BP. The perfect length of the BP ribbon can be determined by

$L_{\mathrm{p}}=N_{\text {unit }} \times L_{0}=\sim 2 \pi \rho$

where $N_{\text {unit }}$ is the number of units along the armchair direction of the BP ribbon. The deformation-based elastic potential energy of the BP ribbon leads to an increase rather than a decrease of the potential energy of the system. For that reason, we choose three BP ribbons of different lengths near different CNTs (see Table 1 ) to reveal the radius/curvature effect on the winding of the BP ribbon.

The second factor is the variation of surface potential energy that depends on the interactions among the curved BP ribbon and the inner and outer CNTs. The value of $\triangle \mathrm{PE}$ due to surface interactions among the three components in system can be expressed as

$\Pi_{\mathrm{vdW}}=\Pi_{\mathrm{I}-\mathrm{BP}}+\Pi_{\mathrm{O}-\mathrm{BP}}+\Pi_{\mathrm{I}-\mathrm{O}}$

where the first part of Eq. (3), i.e., $\Pi_{\mathrm{I}-\mathrm{BP}}$, the interaction between the inner CNT and BP ribbon, is the van der Waals (vdW) interaction energy between CNTs and a BP ribbon. As the width of BP ribbons remains the same ( $w=\sim 2.32 \mathrm{~nm}$ ), the value of $\Pi_{\mathrm{I}-\mathrm{BP}}$ depends only on the length of $\mathrm{BP}$ ribbon. The interaction energy can be obtained by subtracting the total potential energy of the three isolated components from the total potential energy of the system as the distance between two edges of $\mathrm{BP}$ ribbon they attract each other. On the other hand, from our previous study [25,26], curvature of BP ribbon along the armchair direction occurs more easily than that along zigzag direction. Hence, the winding direction would be identical to the armchair direction during winding. This prediction is checked via numerical tests. The second item in Eq. (3) is the interaction energy between the outer CNT and the BP ribbon. At the initial stage of the winding process, we place the ribbon at the center of the inner tube, such that the distance between the BP ribbon and the two outer tubes is $>1.5 \mathrm{~nm}$, i.e., $0.5\left(l_{0}-w\right)>1.5 \mathrm{~nm}$. Therefore, the value of $\Pi_{\mathrm{O}-\mathrm{BP}}$ is initially zero. However, if the BP ribbon attracted on the inner tube moves towards an outer tube, the value of $\Pi_{\mathrm{O}-\mathrm{BP}}$ will not be zero. That change may occur soon after the start of winding of the BP ribbon on a chiral CNT. Both $\Pi_{\mathrm{I}-\mathrm{BP}}$ and $\Pi_{\mathrm{O}-\mathrm{BP}}$ lead to a decrease in the $\triangle \mathrm{PE}$ of the system. The third part of Eq. (3), i.e., $\Pi_{\mathrm{I}-\mathrm{O}}$, represents the interaction between CNTs attributable to the vibration of atoms on CNTs. Because outer CNTs are fixed in simulation, the value of $\Pi_{\mathrm{I}-\mathrm{O}}$ stabilizes quickly after starting the simulation. The value of $\Pi_{\mathrm{I}-\mathrm{O}}$ depends on the temperature T, i.e., the value of $\Pi_{\mathrm{I}-\mathrm{O}}$ is higher at higher temperature.

The final factor is the chemical reaction energy of the BP due to the breakage or generation of $\mathrm{P}-\mathrm{P}$ bonds on the $\mathrm{BP}$ ribbon in simulation. We know that a $\left(s p^{3}\right) \mathrm{P}-\left(s p^{3}\right) \mathrm{P}$ bond is very weak, which leads to instability of the BP at high temperature. During the winding of a BP ribbon on a CNT, the $\mathrm{P}-\mathrm{P}$ bonds on the curved outer surface of the BP ribbon are easily broken, leading to an increase in the $\triangle \mathrm{PE}$ of the system. This should be avoided if we want to obtain a perfect nanotube from BP ribbon. In Fig. 1b, the phosphorus atoms at the edges along the CNT axis are unsaturated. If there is no broken $\mathrm{P}-\mathrm{P}$ bond, and simultaneously the free edges of BP ribbon along the axis of the CNT join together, new $\mathrm{P}-\mathrm{P}$ bonds could be generated. In this situation, the $\triangle \mathrm{PE}$ of the system drops. Mathematically, the chemical reaction energy can be expressed as the total number of new $\mathrm{P}-\mathrm{P}$ bonds, i.e.,

$\Pi_{\text {React }}=N_{\mathrm{nb}} \times \mathrm{d} \Pi_{\mathrm{P}-\mathrm{P}}$

where $N_{\mathrm{nb}}$ can be considered as the variation of the number of $\mathrm{P}-\mathrm{P}$ bonds in BP ribbon during winding. If there is neither broken bond nor new bond generated, $N_{\mathrm{nb}}=0$. As predicted by [39], the potential energy of an edge atom on BP ribbon depends on its position. For the yellow atoms in the BP ribbon shown in Fig. 1, the potential energy of edge atoms is between $[-4.82,-4.76] \mathrm{eV}$ (mean value is $-4.79 \mathrm{eV}$ ). For a bulk atom in BP, the value is $\sim-5.12 \mathrm{eV}$, which is estimated by Stillinger-Weber potential [32]. Hence, the generation of every new $\mathrm{P}-\mathrm{P}$ bond between two layers with edge atoms (red and yellow) in
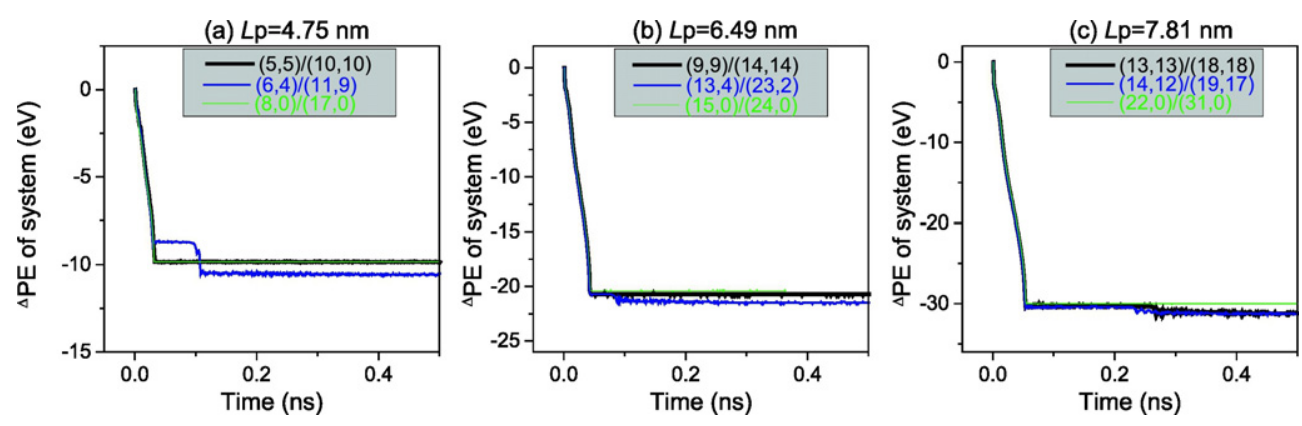

Fig. 2. Histories of $\triangle \mathrm{PE}$ of the system at $8 \mathrm{~K}$ during winding of $\mathrm{BP}$ ribbon on different inner $\mathrm{CNTs}$. 
Table 3

$\triangle \mathrm{PE}$ of the system at $8 \mathrm{~K}$ before and after winding of BP ribbon on different CNTs. $\triangle \mathrm{PE}=\Delta \mathrm{PE} 1+\triangle \mathrm{PE} 2, \Delta \mathrm{PE} 1$ is the first jump of potential energy from the initial peak value to the first plateau, and $\triangle \mathrm{PE} 2$ is the second jump to the minimum. If there is only one jump of $\triangle \mathrm{PE}, \triangle \mathrm{PE} 2=0$. The winding times $(\mathrm{WT})$ with respect to both jumps are labeled WT1 and $\mathrm{WT} 2$, respectively.

\begin{tabular}{lllll}
\hline$L_{\mathrm{p}}$ & $\mathrm{CNTs}$ & Initial PE/eV & $\Delta \mathrm{PE} 1+\Delta \mathrm{PE} 2 / \mathrm{eV}$ & $\mathrm{WT} 1 / \mathrm{WT} 2 / \mathrm{ps}$ \\
\hline \multirow{2}{*}{$4.75 \mathrm{~nm}$} & $(5,5) /(10,10)$ & -5999.0 & $9.85+0$ & $33 / 33$ \\
& $(6,4) /(11,9)$ & -5764.7 & $8.87+1.63$ & $30 / 108$ \\
\multirow{3}{*}{$6.49 \mathrm{~nm}$} & $(8,0) /(17,0)$ & -6592.5 & $9.84+0$ & $32 / 32$ \\
& $(9,9) /(14,14)$ & $-10,326.4$ & $20.50+0$ & $43 / 43$ \\
& $(13,4) /(23,2)$ & $-13,666.3$ & $20.70+0.80$ & $43 / 88$ \\
$7.81 \mathrm{~nm}$ & $(15,0) /(24,0)$ & $-11,436.5$ & $20.40+0$ & $44 / 44$ \\
& $(13,13) /(18,18)$ & $-14,638.6$ & $30.30+0.90$ & $54 / 267$ \\
& $(14,12) /(19,17)$ & $-15,290.7$ & $30.50+0.70$ & $54 / 270$ \\
& $(22,0) /(31,0)$ & $-16,261.6$ & $30.00+0$ & $55 / 55$ \\
\hline
\end{tabular}

the BP leads to potential energy drop of about twice $-0.33 \mathrm{eV}$, i.e., $\mathrm{d} \Pi_{\mathrm{P}-}$ $\mathrm{p}=\sim-0.66 \mathrm{eV}$.

From the analysis above, the deformation-based elastic potential energy is formed from both bonded and non-bonded interaction among not only the atoms on BP ribbon but also the neighbor atoms on CNTs. When deformation of BP ribbon needs more energy than the interaction energy between BP and CNTs (Eq. (3)), BP cannot wind on CNT even if the length of $\mathrm{BP}$ is high enough. On the other hand, if $\mathrm{BP}$ ribbon can be attracted by and tightly upon a CNT, it indicates that the deformation potential energy is far less than the interaction energy among the atoms on both BP ribbon and CNTs. In this case, the variation of the potential energy of the system is able to illustrate the variation process of the configuration of the system.

\section{Results and discussion}

\subsection{Winding of BP ribbon at ultralow temperature, e.g., $8 \mathrm{~K}$}

At $8 \mathrm{~K}$, the $\triangle \mathrm{PE}$ histories of the systems with different components are shown in Fig. 2. When the BP ribbon with $L_{\mathrm{p}}=4.75 \mathrm{~nm}$ is attracted by axially commensurate double walled CNTs, i.e., $(5,5) /(10,10)$ or $(8,0) /(17,0)$, the $\triangle P E$ curves are aligned very well (Fig. 2a). A similar phenomenon can also be found at the earlier stage when $L_{\mathrm{p}}$ is greater (see Fig. 2b, c). During the process, the drop in the potential energy of the system is caused mainly by both vdW interaction and the released energy in generation of new $\mathrm{P}-\mathrm{P}$ bonds on the BP ribbon. From the previous theoretical analysis, the quantity of $\triangle \mathrm{PE}$ of the system is actually greater than that given in Table 3 because of the growth of potential energy for deformation of the BP ribbon $\left(\Pi_{\text {Deform }}\right.$ in Eq. $\left.(1)\right)$ during winding.
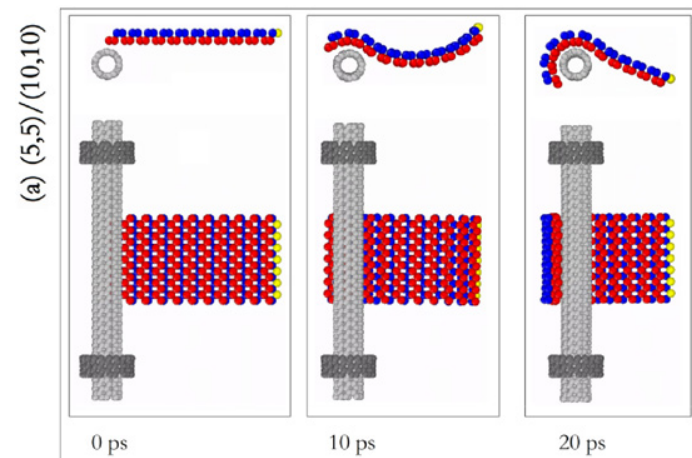

$20 \mathrm{ps}$
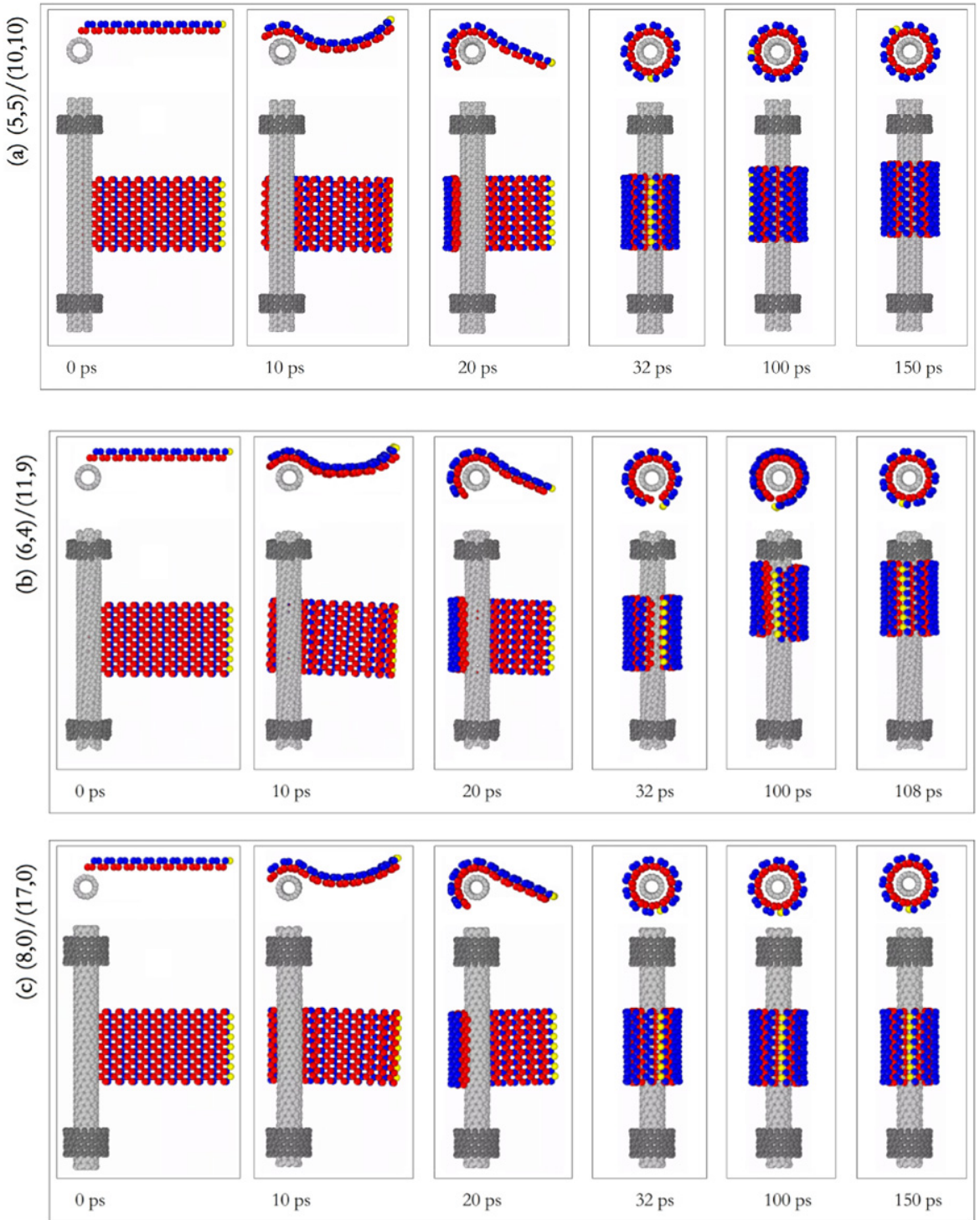

Fig. 3. Snapshots of the winding process of BP ribbon on different inner CNTs at $8 \mathrm{~K}$. 
When the BP ribbon is attracted by incommensurate double walled CNTs, e.g., $(6,4) /(11,9)$, the potential energy of the system usually has two jumps, as can be seen from the configurations of the blue curves in Fig. 2. For example, when the BP ribbon winds on a $(6,4)$ tube, the potential energy of the system first drops due to the interaction of BP ribbon and inner CNT $\left(\Pi_{\mathrm{I}-\mathrm{BP}}\right.$ in Eq. (3)) during the first 30 ps (Table 3 ). After a period of running, the second drop in the potential energy of the system occurs. From Table 3 we know that the value of WT2 with respect to the second drop in potential energy of the system is $108 \mathrm{ps}$. Comparing the configurations of the system from 0 to 108 ps (see group $(6,4) /(11,9)$ in Fig. 3b, and Movie 1$)$, we conclude that the second drop in potential energy of the system, $1.63 \mathrm{eV}$ (Table 3 ) is caused by two features occurring simultaneously, namely generation of new P-P bonds (occurs during 100 and 108 ps) ( $\Pi_{\text {React }}$ in Eq. (4)) and interaction between the BP tube and an outer CNT ( $\Pi_{\mathrm{O}-\mathrm{BP}}$ in Eq. (3)). As $L_{\mathrm{p}}$ is different from $4.75 \mathrm{~ns}$, the second drop in potential energy of the system is no more than $1.0 \mathrm{eV}$. For example, $\Delta \mathrm{PE} 2=0.80 \mathrm{eV}$ when $L_{\mathrm{p}}=$ $6.49 \mathrm{~nm}$ or $\triangle \mathrm{PE} 2=0.70 \mathrm{eV}$ when $\mathrm{L}_{\mathrm{p}}=7.81 \mathrm{~nm}$ (see Table 3). The reason is that the second drop in potential energy of the system is only caused by the attraction between the BP tube and an outer CNT. The fluctuation in the $\triangle \mathrm{PE}$ curve in Fig. $2 \mathrm{~b}$ or $2 \mathrm{c}$ is due to frequent collisions between the BP tube and an outer CNT (Movies 2, 3). The reason for the $\mathrm{BP}$ ribbon sliding towards an outer CNT is that the potential barrier on the inner chiral CNT shows a helix [40]. The relative rotation between the BP ribbon and the inner CNT determines which of the outer CNTs will become closer to the BP ribbon.

When investigating the winding speeds of the BP ribbon on an inner $\mathrm{CNT}$, we find that the same BP ribbons display the same duration of winding on different CNTs. However, with a chiral inner CNT on which the BP ribbon winds, extra time may be involved with the generation of new $\mathrm{P}-\mathrm{P}$ bonds once the CNT goes closer to the BP. For example, after $32 \mathrm{ps}$ the $\mathrm{BP}$ winds on the inner CNT, whereas at 108 ps the two edges of the BP meet and bond perfectly (see group $(6,4) /(11,9)$ in Fig. $3 b$ ).

\subsection{Winding of BP ribbon at temperature $\geq 50 \mathrm{~K}$}

As the temperature increases, the winding process of the BP ribbon on a CNT becomes complex and different from that at $8 \mathrm{~K}$. For example, the $\triangle \mathrm{PE}$ of the system shows a quick jump (circled in Fig. 4a, b \& c-50 K) at the start of winding. The jump is caused by the sharp variation in the configuration of both the CNT and the BP ribbon after being relaxed in a canonical NVT ensemble at a temperature higher than $8 \mathrm{~K}$. The jump also occurs in other cases at a temperature higher than $50 \mathrm{~K}$. Moreover, the variation of the jump is greater at higher temperature.

Observation of the winding process of the BP ribbon on a CNT at temperatures higher than $8 \mathrm{~K}$ shows that fortunately, the BP ribbon can still be perfectly wound on any CNT at $50 \mathrm{~K}$ (see the inserts in Fig. 4a, b \& c-50 K, Movie 4). However, at a temperature higher than $50 \mathrm{~K}$, e.g., at $100 \mathrm{~K}$, the BP ribbon cannot be perfectly wound on such CNTs as $(13,4),(15,0),(13,13),(14,12)$, and $(22,0)$. That means that it is very difficult to wind a BP ribbon on a CNT with larger radius at the same temperature. As we observe the process of the BP ribbon winding on a CNT with a larger radius, e.g., $(15,0)$ (Movie 5) or $(14,12)$ (Movie 6 ), we find that the edge of the BP ribbon near the CNT is first attracted onto the CNT. As well, during the winding process, the free edge of the $\mathrm{BP}$ also moves close to the CNT (at 20 ps in Fig. 5a, b). However, as the overlap between the BP ribbon and CNT increases, the middle part of the $\mathrm{BP}$ ribbon becomes a curve that is opposite to the curving direction of the CNT (at 20 ps in Fig. 5), and simultaneously, the free edge of the $\mathrm{BP}$ keeps moving to the CNT due to the inertia effect. Hence, a critical curvature of the middle part of the BP ribbon develops and is followed by the breakage of $\mathrm{P}-\mathrm{P}$ bonds on the red layer (at 26-27 ps in Fig. 5).
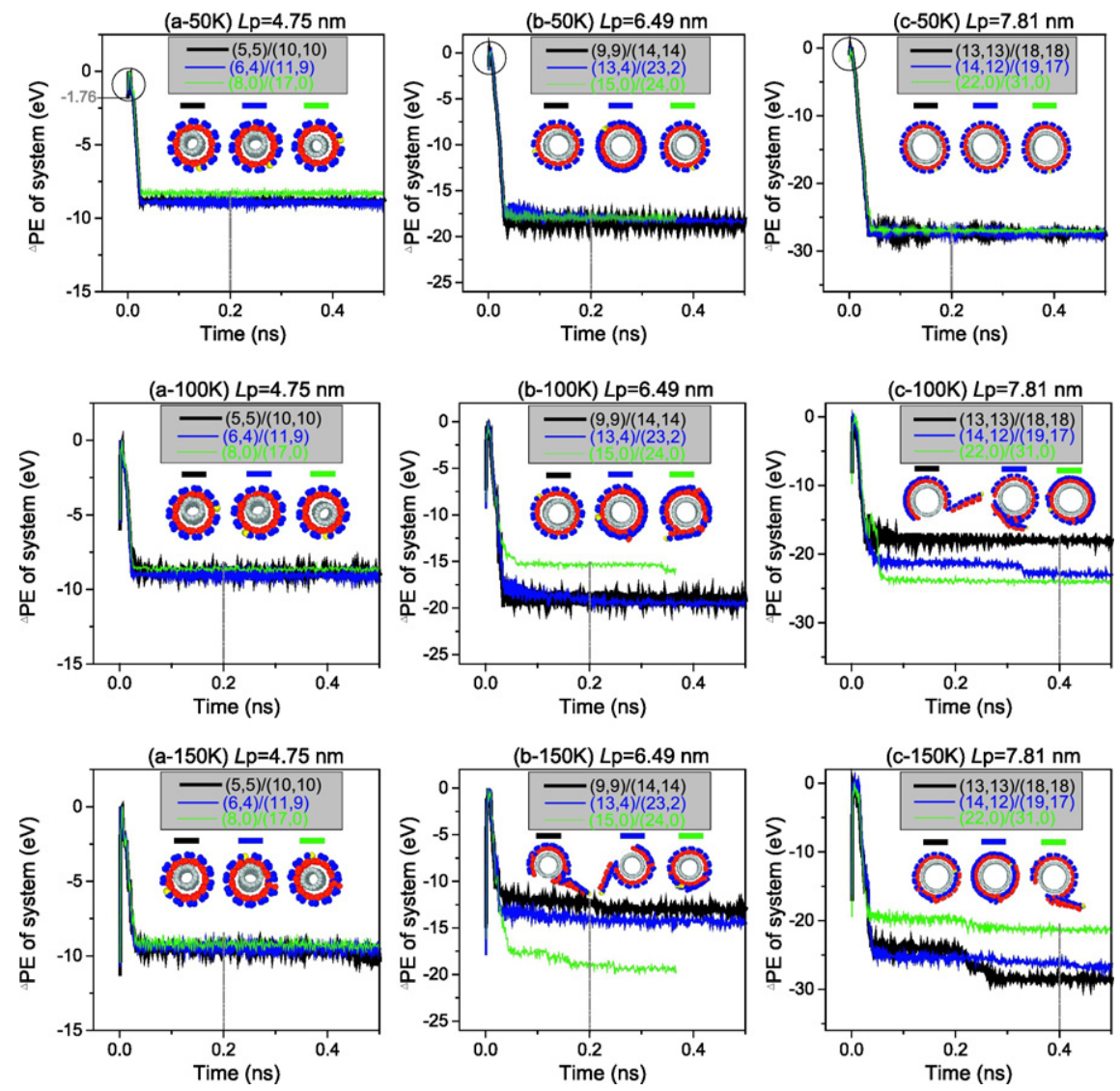

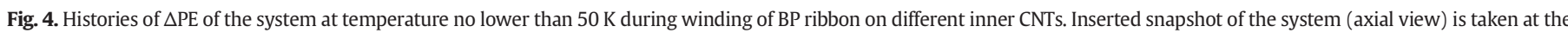
moment indicated by a dashed line. 

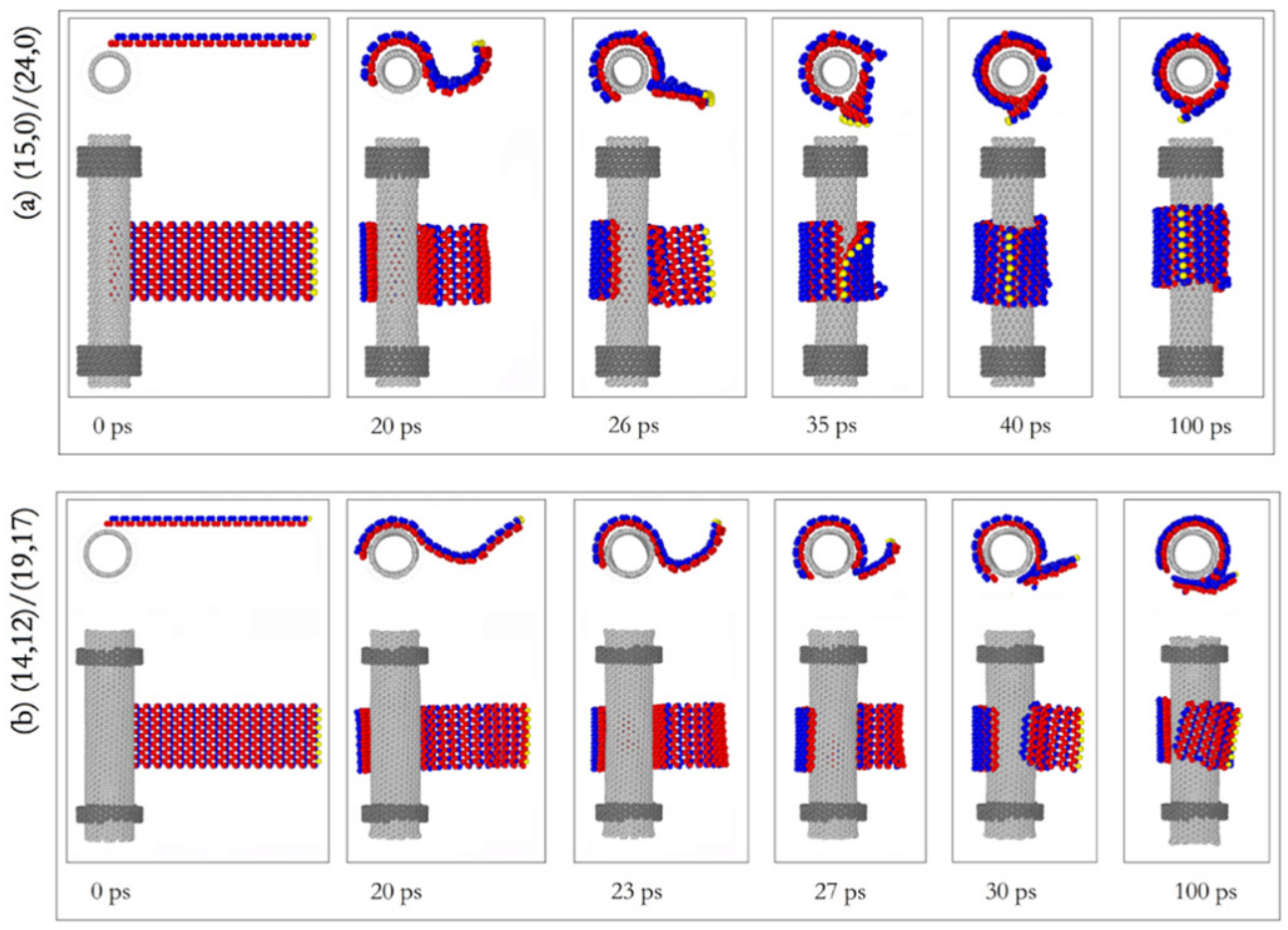

Fig. 5. Snapshots in winding process of BP ribbon on $(15,0)$ or $(14,12)$ CNT at $100 \mathrm{~K}$.

If the length of the BP ribbon is small, e.g., $L_{\mathrm{p}}=6.49 \mathrm{~nm}$, the free part of the BP ribbon will continue to be attracted onto the CNT after the breakage. The reason is that the inertia of the free part is low, i.e., the number of phosphorus atoms is low, and the difference in relative velocity between the two parts of the broken BP ribbon is not great. Hence, the free part is under strong attraction from the CNT as the rest of the BP ribbon rotates together with the CNT. If the length of the BP ribbon is greater, e.g., $L_{\mathrm{p}}=7.81 \mathrm{~nm}$, the BP ribbon will break into two parts due to the higher inertia effect, the free part of the BP ribbon will move closer to CNT, and it also will finally be attracted to the CNT. However, the two parts of the BP ribbon cannot be united into a perfect BP tube (at 100 ps in Fig. 5a, b). Besides the effect of the length of the BP ribbon on the winding process, the chirality of the CNT also influences the winding results. From Fig. 5, we also find that the BP ribbon is attracted symmetrically by a zigzag CNT, causing the motion of the atoms at the free edge of the $\mathrm{BP}$ ribbon to have the same velocity as the CNT. Hence, the broken $\mathrm{P}-\mathrm{P}$ bonds are almost on the same column (along the tube axis or z-direction) and the free part of the BP ribbon can still be attracted almost symmetrically by the CNT (at 40 ps in Fig. 5a). If the CNT is a chiral one, e.g., $(14,12)$, it provides asymmetric attraction to the BP ribbon. The free part of the BP ribbon rotates while moving close to the chiral CNT. The rotation of the free part of the $\mathrm{BP}$ ribbon is caused by the helical potential barrier on the surface of the CNT (at 100 ps in Fig. 5b).

From Fig. 4, we also know that a curve of the $\triangle \mathrm{PE}$ of the system with a lower rate of decrease implies that the free part of the BP ribbon is not attracted onto the CNT. Curves with a higher rate of decrease illustrate that the phosphorus atoms are attracted onto the CNT even if the BP ribbon has broken into several parts.

From the inserts in Fig. 4a, b \& c-150 K, we find that the BP ribbon can be successfully wound on the $(5,5)$ CNT only when the system is at $150 \mathrm{~K}$. It is known that the vibration of atoms on the BP ribbon becomes more marked at $150 \mathrm{~K}$ than at temperatures below $100 \mathrm{~K}$. Strong vibration of phosphorus atoms results in large deformation of the ribbon. As the $\mathrm{P}-\mathrm{P}$ covalent bond is far weaker than the $\mathrm{C}-\mathrm{C}$ bond, it is more (a) At $8 \mathrm{~K}$

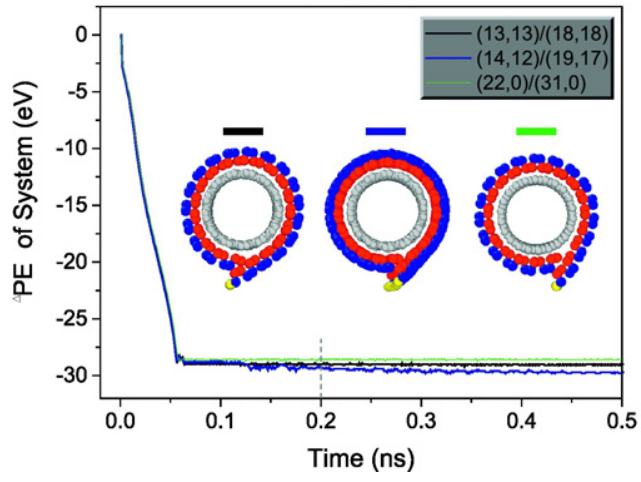

(b) At $50 \mathrm{~K}$

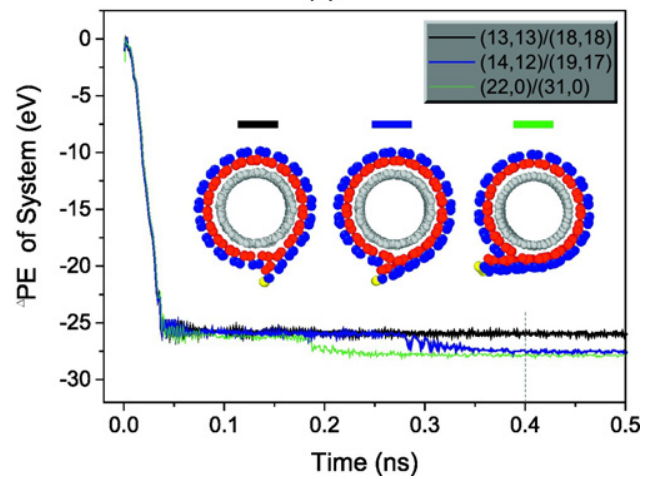

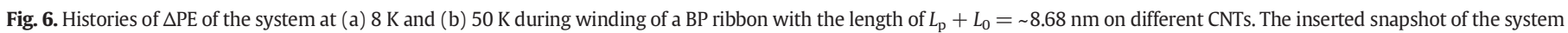
(axial view) is chosen at the moment labeled with a dashed line. 


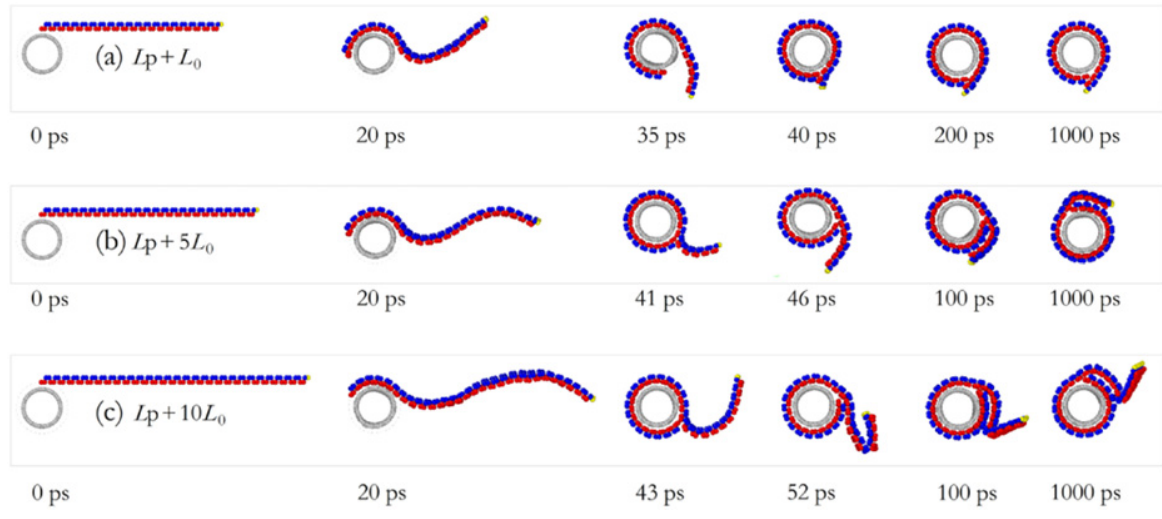

Fig. 7. Snapshots in winding process of BP ribbons with different lengths on CNT $(13,13)$ at $50 \mathrm{~K}$.

easily broken under such large deformation. Taking this reason into consideration, to obtain a perfect BP tube from ribbon wound on a CNT, vibration of the free part of the BP should be reduced.

\subsection{Can a BP ribbon longer than Lp be wound successfully?}

In our previous discussion, we assumed that the length of a BP ribbon was a special value that led to the perfect nanotube from the ribbon, i.e., Eq. (2) $L_{\mathrm{p}}=N_{\text {unit }} \times L_{0}=\sim 2 \pi\left(r+\sigma_{\mathrm{C}-\mathrm{P}}+0.5 d_{\mathrm{BP}}\right)$. Can the ribbon still be perfectly wound onto a nanotube at a very low temperature, e.g., $\leq 50 \mathrm{~K}$, if we choose a BP ribbon with a length greater than $L_{\mathrm{p}}$ ? Here we choose a standard BP ribbon with $L_{\mathrm{p}}=8.24 \mathrm{~nm}$ for comparison. First, we add just one more unit cell along the armchair direction, so that the length of the new BP ribbon is $L_{\mathrm{p}}+L_{0}$, and put it near three different CNTs, i.e., CNT (13,13), (14,12), and (22,0).

Fig. 6 illustrates the $\triangle \mathrm{PE}$ histories of the system during the winding process of the longer BP ribbon at (a) $8 \mathrm{~K}$ and (b) $50 \mathrm{~K}$. It can be seen that most of the curves have only one jump before $100 \mathrm{ps}$. The inserts in Fig. 6 show that the two edges of the BP ribbon along tube axis are not joined, as can be judged easily from the positions of the yellow atoms with respect to the red atoms on the other edge of the BP ribbon. In other words, the free edge (with yellow atoms) overlaps with the other edge rather than attaching to the CNT. It can be concluded that the BP ribbon with length of $\left(L_{\mathrm{p}}+L_{0}\right)$ can be wound onto the CNT but cannot form a perfect $\mathrm{BP}$ nanotube at the temperature of $\mathrm{T} \leq 50 \mathrm{~K}$.

On the other hand, we also try to wind a BP ribbon of length $L_{\mathrm{p}}+5 L_{0}$ or $L_{\mathrm{p}}+10 L_{0}$ onto a CNT $(13,13)$. From the snapshots in Fig. 7 , it can be seen that the free edge of the BP ribbon has a different configuration in the final state. For example, when the length of the BP ribbon is $L p+5 L_{0}$, the free edge finally overlaps with the other edge of the ribbon, and the BP ribbon becomes a nanoscroll. However, if the BP ribbon is longer, e.g., with the length of $L_{\mathrm{p}}+10 L_{0}$, the free edge of the BP ribbon may be distant from the inner CNT. The reason is that the blue phosphorus atoms near the broken $\mathrm{P}-\mathrm{P}$ bonds attract one another and the atoms lack sufficient kinetic energy to change the configuration further. From these results, we conclude that a BP ribbon with a length longer than $L_{\mathrm{p}}$ may be wound, but cannot form a perfect BP nanotube even at very low temperature.

\section{Conclusions}

Using a molecular dynamics simulation approach, we investigated the possibility of forming a nanotube from BP nanoribbon via winding on a CNT. After a series of discussions of the variations of configuration of a BP ribbon in the winding process at very low temperature, the following six conclusions can be drawn. First, during the winding process, the potential energy of the system decreases due to both vdW interaction among components in the system and released energy in the generation of new $\mathrm{P}-\mathrm{P}$ bonds on the $\mathrm{BP}$ ribbon. A greater decrease of potential energy leads to a more stable configuration of the system. Second, if the two edges of a BP ribbon along the tube axis do not join soon after the BP ribbon is attracted onto the inner tube, the history curve of the $\triangle \mathrm{PE}$ of the system contains at least two sharp decreases. The first jump is attributed to the interaction between the BP ribbon and the inner CNT. The second jump could be caused by the generation of new $\mathrm{P}-\mathrm{P}$ bonds. If the jump occurs after formation of a perfect $\mathrm{BP}$ tube, it is mainly due to the BP tube being attracted by an outer CNT. Third, a $\mathrm{BP}$ nanotube can be formed on the condition that the BP ribbon has perfect length, i.e., $L \mathrm{p}=N_{\text {unit }} \times L_{0}=\sim 2 \pi\left(r+\sigma_{\mathrm{C}-\mathrm{P}}+0.5 d_{\mathrm{BP}}\right)$, and the temperature is very low, e.g., $\leq 50 \mathrm{~K}$. Fourth, at $8 \mathrm{~K}$, the formation of a perfect nanotube from the same BP ribbon (with perfect length) on a chiral inner CNT takes longer because extra time is required for the generation of new $\mathrm{P}-\mathrm{P}$ bonds after the $\mathrm{BP}$ ribbon has been compactly attracted by the CNT. Fifth, at higher temperatures, e.g., $\geq 50 \mathrm{~K}$, even with perfect length, it is difficult to perfectly wind a BP ribbon on a CNT with higher radius. That task becomes possible if the vibration of the free part of the $\mathrm{BP}$ ribbon is reduced. Final, if the length of a BP ribbon is greater than the perfect length, the ribbon cannot form a perfect BP nanotube even at very low temperature.

In the present study, defect-free CNTs are considered. If there is an unsaturated carbon atom in the CNT existing nearby the BP nanotube, chemical reaction between the unsaturated carbon atom and a phosphorus atom on the BP nanotube may happen, which will lead to failure of fabrication of an ideal BP nanotube. Fortunately, an ideal CNT can be easily obtained, and the present simulations are reasonable for the guidance of future fabrication of BP nanotubes.

Supplementary data to this article can be found online at http://dx. doi.org/10.1016/j.matdes.2017.02.084.

\section{References}

[1] K.S. Novoselov, A.K. Geim, S.V. Morozov, D. Jiang, Y. Zhang, S.V. Dubonos, I.V. Grigorieva, A.A. Firsov, Electric field effect in atomically thin carbon films, Science 306 (5696) (2004) 666-669.

[2] C. Lee, X. Wei, J.W. Kysar, J. Hone, Measurement of the elastic properties and intrinsic strength of monolayer graphene, Science 321 (5887) (2008) 385-388.

[3] D.S. Li, W.Z. Wu, X.L. Zhou, D.W. Zuo, S.Q. Lu, Y.B. Gao, Q.H. Qin, Microstructure and mechanical properties of graphene/Al composites, Zhongguo Youse Jinshu Xuebao 25 (6) (2015) 1498-1504

[4] K.S. Kim, Y. Zhao, H. Jang, S.Y. Lee, J.M. Kim, K.S. Kim, J.-H. Ahn, P. Kim, J.-Y. Choi, B.H. Hong, Large-scale pattern growth of graphene films for stretchable transparent electrodes, Nature 457 (7230) (2009) 706-710.

[5] D.C. Elias, R.R. Nair, T. Mohiuddin, S. Morozov, P. Blake, M. Halsall, A. Ferrari, D. Boukhvalov, M. Katsnelson, A. Geim, Control of graphene's properties by reversible hydrogenation: evidence for graphane, Science 323 (5914) (2009) 610-613.

[6] Y. Li, Z. Zhou, S. Zhang, Z. Chen, $\mathrm{MoS}_{2}$ nanoribbons: high stability and unusual electronic and magnetic properties, J. Am. Chem. Soc. 130 (49) (2008) 16739-16744.

[7] R. Sundaram, M. Engel, A. Lombardo, R. Krupke, A. Ferrari, P. Avouris, M. Steiner, Electroluminescence in single layer $\mathrm{MoS}_{2}$, Nano Lett. 13 (4) (2013) 1416-1421.

[8] K. Suenaga, C. Colliex, N. Demoncy, A. Loiseau, H. Pascard, F. Willaime, Synthesis of nanoparticles and nanotubes with well-separated layers of boron nitride and carbon, Science 278 (5338) (1997) 653-655. 
[9] A. Pakdel, C. Zhi, Y. Bando, D. Golberg, Low-dimensional boron nitride nanomaterials, Mater. Today 15 (6) (2012) 256-265.

[10] L. Li, Y. Yu, G.J. Ye, Q. Ge, X. Ou, H. Wu, D. Feng, X.H. Chen, Y. Zhang, Black phosphorus field-effect transistors, Nat. Nanotechnol. 9 (5) (2014) 372-377.

[11] S. Zhang, J. Yang, R. Xu, F. Wang, W. Li, M. Ghufran, Y.-W. Zhang, Z. Yu, G. Zhang, Q.H. Qin, Extraordinary photoluminescence and strong temperature/angle-dependent Raman responses in few-layer phosphorene, ACS Nano 8 (9) (2014) 9590-9596.

12 W. Qiu, Q. Li, Z.K. Lei, Q.H. Qin, W.L. Deng, Y.L. Kang, The use of a carbon nanotube sensor for measuring strain by micro-Raman spectroscopy, Carbon 53 (2013) 161-168.

[13] W. Qiu, Y.L. Kang, Z.K. Lei, Q.H. Qin, Q. Li, A new theoretical model of a carbon nanotube strain sensor, Chin. Phys. Lett. 26 (8) (2009) 080701.

[14] W. Qiu, Y.L. Kang, Z.K. Lei, Q.H. Qin, Q. Li, Q. Wang, Experimental study of the Raman strain rosette based on the carbon nanotube strain sensor, J. Raman Spectrosc. 41 (10) (2010) 1216-1220.

[15] N. Khani, M. Yildiz, B. Koc, Elastic properties of coiled carbon nanotube reinforced nanocomposite: a finite element study, Mater. Des. 109 (2016) 123-132.

[16] C. Liu, H. Shi, H. Yang, S. Yan, S. Luan, Y. Li, M. Teng, A.F. Khan, J. Yin, Fabrication of antibacterial electrospun nanofibers with vancomycin-carbon nanotube via ultrasonication assistance, Mater. Des. 120 (2017) 128-134.

[17] Y. Takao, A. Morita, Electronic structure of black phosphorus: tight binding approach, Physica B + C 105 (1-3) (1981) 93-98.

[18] H. Asahina, K. Shindo, A. Morita, Electronic structure of black phosphorus in selfconsistent pseudopotential approach, J. Phys. Soc. Jpn. 51 (4) (1982) 1193-1199.

[19] J. Qiao, X. Kong, Z.-X. Hu, F. Yang, W. Ji, High-mobility transport anisotropy and linear dichroism in few-layer black phosphorus, Nat. Commun. 5 (2014) 4475.

[20] M. Buscema, D.J. Groenendijk, G.A. Steele, H.S. van der Zant, A. Castellanos-Gomez, Photovoltaic effect in few-layer black phosphorus PN junctions defined by local electrostatic gating, Nat. Commun. 5 (2014) 4651.

[21] J.-W. Jiang, H.S. Park, Mechanical properties of single-layer black phosphorus, J. Phys. D. Appl. Phys. 47 (38) (2014) 385304.

[22] Q. Wei, X. Peng, Superior mechanical flexibility of phosphorene and few-layer black phosphorus, Appl. Phys. Lett. 104 (25) (2014) 251915.

[23] Z.-D. Sha, Q.-X. Pei, Z. Ding, J.-W. Jiang, Y.-W. Zhang, Mechanical properties and fracture behavior of single-layer phosphorene at finite temperatures, J. Phys. D. Appl. Phys. 48 (39) (2015) 395303.

[24] H. Guo, N. Lu, J. Dai, X. Wu, X.C. Zeng, Phosphorene nanoribbons, phosphorus nanotubes, and van der Waals multilayers, J. Phys. Chem. C 118 (25) (2014) 14051-14059.
[25] K. Cai, J. Wan, N. Wei, H. Cai, O.H. Qin, Thermal stability of a free nanotube from single-layer black phosphorus, Nanotechnology 27 (23) (2016) 235703.

[26] K. Cai, J. Wan, N. Wei, Q.H. Qin, Strength and stability analysis of a single-walled black phosphorus tube under axial compression, Nanotechnology 27 (27) (2016) 275701.

[27] A. Kis, K. Jensen, S. Aloni, W. Mickelson, A. Zettl, Interlayer forces and ultralow sliding friction in multiwalled carbon nanotubes, Phys. Rev. Lett. 97 (2) (2006) 025501.

[28] C. Zhang Z Peng S. Chen, Chirality-controlled carbon nanotubes fabricated by selfassembly of graphene nanoribbons, J. Phys. Chem. C 118 (33) (2014) 19477-19483.

[29] H. Yin, K. Cai, Unwinding of a carbon nanoscroll due to high speed rotation, AIP Adv. 5 (10) (2015) 107202

[30] K. Cai, J. Yu, J. Wan, H. Yin, J. Shi, Q.H. Qin, Configuration jumps of rotor in a nanomotor from carbon nanostructures, Carbon 101 (2016) 168-176.

[31] F.H. Stillinger, T.A. Weber, Computer simulation of local order in condensed phases of silicon, Phys. Rev. B 31 (8) (1985) 5262

[32] J.-W. Jiang, Parametrization of Stillinger-Weber potential based on valence force field model: application to single-layer $\mathrm{MoS}_{2}$ and black phosphorus, Nanotechnology 26 (31) (2015) 315706

[33] J.-W. Jiang, T. Rabczuk, H.S. Park, A Stillinger-Weber potential for single-layered black phosphorus, and the importance of cross-pucker interactions for a negative Poisson's ratio and edge stress-induced bending, Nanoscale 7 (14) (2015) 6059-6068.

[34] S.J. Stuart, A.B. Tutein, J.A. Harrison, A reactive potential for hydrocarbons with intermolecular interactions, J. Chem. Phys. 112 (14) (2000) 6472-6486.

[35] J.E. Jones, On the determination of molecular fields. II. From the equation of state of a gas, Proceedings of the Royal Society of London A: Mathematical, Physical and Engineering Sciences, The Royal Society 1924, pp. 463-477.

[36] S. Plimpton, Fast parallel algorithms for short-range molecular dynamics, J. Comput. Phys. 117 (1) (1995) 1-19.

[37] LAMMPS, Molecular dynamics simulator, http://lammps.sandia.gov 2016

[38] S. Nosé, A unified formulation of the constant temperature molecular dynamics methods, J. Chem. Phys. 81 (1) (1984) 511-519.

[39] V. Sorkin, Y. Zhang, The structure and elastic properties of phosphorene edges, Nanotechnology 26 (23) (2015) 235707.

[40] A.M. Popov, I.V. Lebedeva, A.A. Knizhnik, Y.E. Lozovik, B.V. Potapkin, Ab initio study of edge effect on relative motion of walls in carbon nanotubes, J. Chem. Phys. 138 (2) (2013) 024703. 